\title{
Some Results on Greedy Embeddings in Metric Spaces
}

\author{
Tom Leighton • Ankur Moitra
}

Received: 2 March 2009 / Revised: 21 September 2009 / Accepted: 30 September 2009 /

Published online: 20 October 2009

(C) Springer Science+Business Media, LLC 2009

\begin{abstract}
Geographic Routing is a family of routing algorithms that uses geographic point locations as addresses for the purposes of routing. Such routing algorithms have proven to be both simple to implement and heuristically effective when applied to wireless sensor networks. Greedy Routing is a natural abstraction of this model in which nodes are assigned virtual coordinates in a metric space, and these coordinates are used to perform point-to-point routing.

Here we resolve a conjecture of Papadimitriou and Ratajczak that every 3connected planar graph admits a greedy embedding into the Euclidean plane. This immediately implies that all 3-connected graphs that exclude $K_{3,3}$ as a minor admit a greedy embedding into the Euclidean plane. We also prove a combinatorial condition that guarantees nonembeddability. We use this result to construct graphs that can be greedily embedded into the Euclidean plane, but for which no spanning tree admits such an embedding.
\end{abstract}

Keywords Greedy embedding · Papadimitriou-Ratajczak conjecture · Christmas cactus graph $\cdot$ Excluded minor

\section{Introduction}

\subsection{Background}

The study of routing has a long and rich history. But for many important classes of routing problems, routing schemes that are both simple and provably effective have

The research of A. Moitra was supported in part by an MIT (Akamai) Presidential Fellowship.

\section{T. Leighton}

Math. Department and CSAIL, Massachusetts Institute of Technology, Cambridge, MA 02139, USA

e-mail: ftl@math.mit.edu

A. Moitra ( $\triangle)$

EECS Department and CSAIL, Massachusetts Institute of Technology, Cambridge, MA 02139, USA

e-mail: moitra@mit.edu 
so far been elusive. In particular, scalable wireless sensor networks require point-topoint communication, but such ad hoc networks admit no global hierarchical addressing scheme, and there are still no broadly accepted, scalable point-to-point routing schemes despite numerous proposals. Geographic Routing is a family of routing algorithms that uses geographic point locations as addresses for the purposes of routing. Such routing algorithms have proven to be both simple to implement and heuristically effective when applied to wireless sensor networks.

Recent work on routing protocols for wireless sensor networks [2, 7] has focused particular attention on a class of "greedy" algorithms wherein a packet at a node $u$ that is destined for a node $v$ is simply forwarded to any neighbor $u^{\prime}$ of $u$ for which $d\left(u^{\prime}, v\right)<d(u, v)$, where $d(x, y)$ is the Euclidean distance between the locations of $x$ and $y$ in the plane. For such an algorithm to guarantee delivery, it must be the case that for every $u$ and $v$, such a $u^{\prime}$ exists (i.e., that wherever a packet is in the network, there is always a next hop that gets the packet closer in Euclidean distance to its ultimate destination).

Rao et al. [10] proposed a natural abstraction of this model in which nodes are assigned virtual coordinates in a metric space, and these coordinates are used to perform point-to-point routing.

Definition 1 A graph $G=(V, E)$ is said to have a greedy embedding into a metric space $(X, d)$ if there is a function $f: V \rightarrow X$ such that for every pair of distinct nodes $u, v \in V$, there exists a neighbor $u^{\prime}$ of $u$ in $G$ such that $d\left(f\left(u^{\prime}\right), f(v)\right)<$ $d(f(u), f(v))$.

Papadimitriou and Ratajczak [9] considered the case where $(X, d)$ is the Euclidean plane and gave simple examples of graphs which have a greedy embedding (e.g., Hamiltonian graphs) and graphs that admit no greedy embedding into the Euclidean plane (e.g., $K_{r, 6 r+1}$ ). Papadimitriou and Ratajczak conjectured that all 3vertex-connected planar graphs admit a greedy embedding into the Euclidean plane. Throughout this paper, we will be interested only in vertex connectivity, so we will shorten $k$-vertex-connected to $k$-connected.

Conjecture 1 [9] All 3-connected planar graphs admit a greedy embedding into the Euclidean plane.

Papadimitriou and Ratajczak [9] proved that all 3-connected planar graphs admit a greedy-type embedding in 3-dimensions, although for their result, the notion of a greedy embedding is slightly different than the standard definition because the distance function they use is not a metric. Kleinberg [8] considered the case in which $(X, d)$ is the hyperbolic plane and showed that every tree (and consequently every graph) has a greedy embedding in the hyperbolic plane. Dhandapani [3] recently proved that all triangulated 3-connected planar graphs admit a greedy embedding into the Euclidean plane; this is a relaxation of the Papadimitriou-Ratajczak Conjecture. His proof made use of Schnyder Realizers, and used the geometric properties of Schnyder Drawings to find a greedy embedding. 
Eppstein and Goodrich [4] considered the problem of finding a greedy embedding that can be represented succinctly. Many theoretical results on greedy embeddings (including those presented here) require the bit complexity of representing the greedy embedding to exceed the bit complexity of describing the adjacency matrix of the graph. In [4], Eppstein and Goodrich are able to support greedy routing (in hyperbolic space) but substantially improve the bit-complexity of representing the virtual coordinates used by the algorithm. Additionally, Goorich and Strash [6] presented variants of some embeddings given here and were able to obtain similar improvements in the case of greedy embeddings into the Euclidean plane.

\subsection{Our Results}

Here we resolve the Papadimitriou-Ratajczak Conjecture. In fact, we construct a greedy embedding into the Euclidean plane for all circuit graphs (which generalize 3-connected planar graphs). We can apply a theorem in [9] which states that if a 3-connected graph $G$ does not contain $K_{3,3}$ as a minor, then $G$ contains a spanning 3 -connected planar subgraph. If any spanning subgraph of $G$ admits a greedy embedding (into a particular metric space $(X, d)$ ), then $G$ also admits a greedy embedding into this metric space. So this immediately implies that all 3-connected graphs that exclude $K_{3,3}$ as a minor admit a greedy embedding into the Euclidean plane.

Additionally, we provide the first examples of graphs of degree at most 3 that admit no such embedding. We also prove a combinatorial condition that guarantees nonembeddability, and this condition provides a certificate for nonembeddability that can be verified in linear time. We use this result to construct graphs that can be greedily embedded into the Euclidean plane, but for which no spanning tree admits such an embedding.

Perhaps of independent interest, we make use of a decomposition theorem due to Gao and Richter [5]. This is, to the best of our knowledge, this decomposition theorem's first use in theoretical computer science. We use this theorem to find a spanning subgraph that can be greedily embedded into the Euclidean plane, and we believe that this technique can be generally applicable for finding particular types of spanning subgraphs in circuit graphs as needed.

\section{A Greedy Embedding for Circuit Graphs}

Circuit graphs are a relaxation of 3-connected planar graphs. In this section, we prove that all circuit graphs contain a spanning Christmas cactus graph and provide a polynomial-time algorithm to find such a spanning subgraph. We then construct a greedy embedding into the Euclidean plane for all Christmas cactus graphs. This proves the Papadimitriou-Ratajczak Conjecture. As a corollary, any 3-connected graph that excludes $K_{3,3}$ as a minor admits a greedy embedding into the Euclidean plane. 

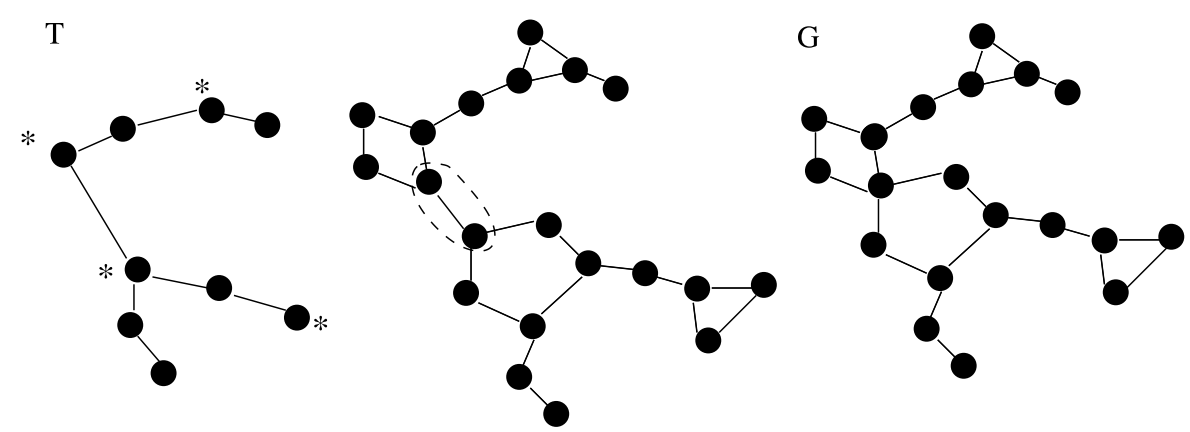

Fig. 1 A Christmas cactus graph $G$ generated by the tree $T$. The nodes in $U$ are denoted with a $*$, and the edge in $F$ is circled

\subsection{Christmas Cactus Graphs}

A cactus graph is a graph for which every edge is part of at most one cycle. In what follows, we will be interested in a special type of cactus graph that we call a Christmas cactus graph.

Definition 2 A Christmas cactus graph $G=(V, E)$ is a connected cactus graph for which the removal of any node $v \in V$ disconnects $G$ into at most two components.

It is well known that a cactus graph can be constructed from a tree by replacing edges with cycles of arbitrary size. Similarly, a Christmas cactus graph can be constructed from a tree by replacing nodes with cycles and contracting edges that are not in cycles. It is easy to see that we can alternatively define a Christmas cactus graph as any graph that can be generated by the following procedure:

Step 1. Let $T$ be a tree, and let $U$ be any subset of nodes of $T$ that contains every node with degree at least 3 in $T$.

Step 2. (Repeat) For each node $u \in U$, replace $u$ with a cycle $C_{u}$ of arbitrary length so that all neighbors of $u$ (in the current graph) are connected by an edge to a distinct node in $C_{u}$.

Step 3. Let $F$ be any set of edges in the graph resulting from Step 2 which are not contained in a cycle.

Step 4. Contract out the edges in $F$.

For example, these steps are shown in Fig. 1.

Simple cycles in a Christmas cactus graph are not necessarily node-disjoint because a path connecting two node-disjoint cycles can be contracted. However, for any two simple cycles $C_{1}$ and $C_{2},\left|C_{1} \cap C_{2}\right| \leq 1$ and for any three simple cycles $C_{1}, C_{2}$, and $C_{3},\left|C_{1} \cap C_{2} \cap C_{3}\right|=0$.

\subsection{Circuit Graphs}

Barnette [1] introduced the class of graphs known as circuit graphs, which he defined to be graphs obtained by deleting a vertex from a 3-connected planar graph. Circuit 
Fig. 2 Obtaining a spanning closed 2-walk from a spanning Christmas cactus graph

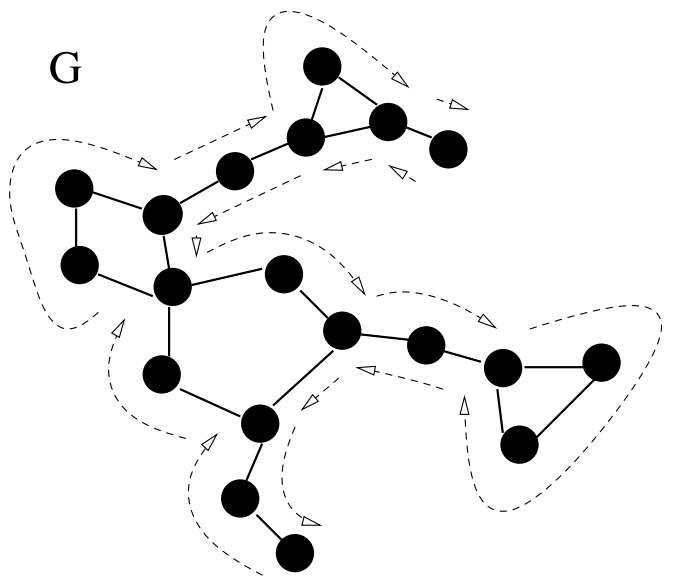

graphs are a relaxation of 3-connected planar graphs but provide a more convenient class of graphs on which to construct inductive proofs. Gao and Richter [5] proved rich structural theorems about the class of circuit graphs and used these results to inductively prove that all circuit graphs contain a spanning closed 2 -walk, i.e., a walk that starts and ends at some vertex $u$ and visits all vertices in $G$ at least once and at most twice. Here we will use these structural results to prove that any 3-connected planar graph (or more generally any circuit graph) contains a spanning Christmas cactus graph. We will then give a greedy embedding for any Christmas cactus graph into the Euclidean plane, and this will prove the Papadimitriou-Ratajczak Conjecture.

We take a short detour to provide intuition as to why the notion of a greedy embedding into the Euclidean plane should be at all related to the existence of a spanning closed 2 -walk. We note that any graph that contains a Hamiltonian cycle also admits a greedy embedding into the Euclidean plane. The reason is that if any spanning subgraph of $G$ admits a greedy embedding into the Euclidean plane, then $G$ also admits a greedy embedding into the Euclidean plane. Also, any cycle admits a greedy embedding into the Euclidean plane, and this implies the claim.

However, not all 3-connected planar graphs contain a Hamiltonian cycle. So we cannot hope to prove the Papadimitriou-Ratajczak Conjecture by finding a Hamiltonian cycle. A spanning closed 2-walk is a relaxation of a Hamiltonian cycle, and such a walk still maintains almost enough structure so that we can reconstruct a greedy embedding into the Euclidean plane from such a walk.

We can consider a spanning Christmas cactus graph to be a type of spanning closed 2-walk:

Claim 1 Any graph $G$ that contains a spanning Christmas cactus graph also contains a spanning closed 2-walk.

Moreover, such a walk can be obtained by performing a depth first search of the tree $T$ that generated the spanning Christmas cactus graph; see Fig. 2.

So here we will use the results of Gao and Richter [5] to prove the existence of a particular type of spanning closed 2-walk-a spanning Christmas cactus graph-in 
every circuit graph. From this spanning subgraph we will construct a greedy embedding into the Euclidean plane. Gao and Richter equivalently define a circuit graph as follows:

Definition 3 A circuit graph is an ordered pair $(G, C)$ such that:

1. $G$ is 2-connected, and $C$ is a polygon in $G$.

2. There is a noncrossing embedding of $G$ in the plane such that $C$ bounds an infinite face.

3. If $(H, K)$ is a 2 -separation ${ }^{1}$ of $G$, then $C \nsubseteq H$ and $C \nsubseteq K$.

A 3-connected planar graph is a circuit graph because $G$ can be embedded using Tutte's rubber band embedding and any 2-separation $(H, K)$ such that $C \subset H$ would imply that $G$ is not 3-connected. We next review some key structural properties of circuit graphs:

Lemma 1 [5] Let $(G, C)$ be a circuit graph embedded in the plane (such that no edges are crossing), and let $C_{1}$ be any polygon in $G$. Then the subgraph $G_{1}$ containing $C_{1}$ and all nodes and edges inside $C_{1}$ (in the plane embedding) is a circuit graph.

Definition 4 [5] A connected graph $G$ is a chain of blocks if each block of $G$ contains at most two cut vertices and each cut vertex ${ }^{2}$ lies in exactly two blocks. Then a chain of blocks can be written as $B_{1}, b_{1}, B_{2}, \ldots, b_{k-1}, B_{k}$ such that the common vertex of blocks $B_{i}$ and $B_{i+1}$ is $b_{i}$. Then a plane chain of blocks is a chain of blocks and a plane embedding such that for all $j, \bigcup_{i \neq j} B_{i}$ is in the infinite face of $B_{j}$.

Because each cut vertex is in exactly two blocks, each $b_{i}$ must be distinct. A block is called trivial if the block is just the edge $b_{i}, b_{i+1}$. Then the seminal work of Gao and Richter gives the following structural result for circuit graphs (see Fig. 3).

Theorem 1 [5] Let $(G, C)$ be a circuit graph, and let $x, y \in C$ be distinct. Then there exists a partition of $V(G)-V(C)$ into $V_{1}, V_{2}, \ldots, V_{m}$ and distinct vertices $v_{1}, v_{2}, \ldots, v_{m} \in V(C)-\{x, y\}$ such that the graph induced by $V_{i} \cup\left\{v_{i}\right\}$ is a plane chain of blocks $B_{i, 1}, b_{i, 1}, \ldots, b_{i, k-1}, B_{i, k}$ such that $v_{i} \in V\left(B_{i, 1}\right)-b_{i, 1}$ and each nontrivial block $B_{i, j}$ has an outer polygon $C_{i, j}$ such that $\left(B_{i, j}, C_{i, j}\right)$ is a circuit graph. ${ }^{3}$

Gao and Richter use this structural result to find a spanning closed 2-walk that visits $x, y$ only once, in any circuit graph by induction. In what follows, we use this

\footnotetext{
${ }^{1} \mathrm{~A} k$-separation of a graph $H=(V, E)$ is a pair $H_{1}, H_{2}$ of edge disjoint subgraphs of $H$, each with at least $k+1$ vertices, such that $H=H_{1} \cup H_{2}$ and $\left|V\left(H_{1}\right) \cap V\left(H_{2}\right)\right|=k$.

${ }^{2} \mathrm{~A}$ cut vertex is a vertex that when removed from $G$, disconnects the graph.

${ }^{3}$ Gao and Richter actually state that $B_{i, j}$ is a block, but from the construction of this block in the proof of the theorem it is clear that $B_{i, j}$ is a subgraph containing all nodes and edges inside (and including) a polygon $C_{i, j}$ in $G$. This implies that $\left(B_{i, j}, C_{i, j}\right)$ is a circuit graph, and Gao and Richter explicitly state this when actually invoking the structural theorem to prove that all circuit graphs contain a closed, spanning 2-walk.
} 
Fig. 3 A plane chain of blocks

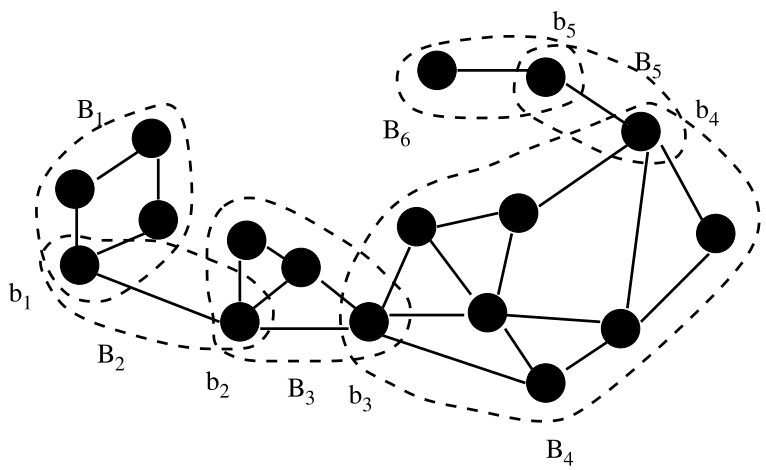

structural result to find a spanning subgraph in any circuit graph that can be greedily embedded in the plane. We will use $\delta_{G}(x)$ to denote the degree of $x$ in $G$. The proof of this theorem given by Gao and Richter is constructive and can be used to find such a decomposition in polynomial time, given the planar drawing of the circuit graph.

Theorem 2 For any circuit graph $(G, C)$ and distinct $x, y \in C$, there exists a Christmas cactus graph $T(G)$ that spans $G$ such that $x, y$ are jointly in a cycle and $\delta_{T(G)}(x)=\delta_{T(G)}(y)=2$, and such a subgraph can be found in polynomial time.

Proof The proof is by induction on the number of vertices in the circuit graph $(G, C)$. Let $x, y \in V(C)$ be distinct. Applying Theorem 1, there exists a partition of $V(G)-V(C)$ into $V_{1}, V_{2}, \ldots, V_{m}$ and distinct vertices $v_{1}, v_{2}, \ldots, v_{m} \in$ $V(C)-\{x, y\}$ such that the graph induced by $V_{i} \cup\left\{v_{i}\right\}$ is a plane chain of blocks $B_{i, 1}, b_{i, 1}, \ldots, b_{i, k-1}, B_{i, k}, v_{i} \in V\left(B_{i, 1}\right)-b_{i, 1}$ and each nontrivial block $B_{i, j}$ has an outer polygon $C_{i, j}$ such that $\left(B_{i, j}, C_{i, j}\right)$ is a circuit graph.

By the definition of a plane chain of blocks, each cut vertex $b_{i, k}$ must be distinct. The chain of blocks $B_{i, 1}, b_{i, 1}, \ldots, b_{i, k-1}, B_{i, k}$ is a plane chain of blocks w.r.t. the original plane embedding. $v_{i}$ is on the infinite face in $G$, and this implies that $v_{i} \in C_{i, 1}$ because $v_{i}$ must be in the infinite face in $\left(B_{i, 1}, C_{i, 1}\right)$. Choose $b_{i, 0}=v_{i}$ and $b_{i, k}$ to be any vertex $\in B_{i, k}-b_{i, k-1}$ that is contained in the infinite face $C_{i, k}$. If $B_{i, k}$ is a trivial block, then just choose $b_{i, k}$ to be the remaining endpoint. Directly from the structural theorem, $v_{i} \in B_{i, 1}-b_{i, 1}$. Then each $b_{i, j}$ is distinct, and contained in both infinite faces $C_{i, j}$ and $C_{i, j+1}$.

By induction each (nontrivial) $B_{i, j}$ has a Christmas cactus spanning subgraph such that $b_{i, j-1}, b_{i, j}$ are jointly on a simple cycle and $\delta_{T\left(B_{i, j}\right)}\left(b_{i, j-1}\right), \delta_{T\left(B_{i, j}\right)}\left(b_{i, j}\right)=2$. Then join the spanning Christmas cactus graph of $B_{i, j}$ to the spanning Christmas cactus graph of $B_{i, j+1}$ by joining $b_{i, j}$ in each spanning Christmas cactus graph by an edge, and contracting the edge.

If the neighboring block in the plane chain is a trivial block, then just connect a nontrivial block to the next nontrivial block by a path of the trivial blocks. If $B_{i, 1}$ is not trivial, then the result is a Christmas cactus spanning subgraph for the plane chain of blocks $B_{i, 1}, b_{i, 1}, \ldots, b_{i, k-1}, B_{i, k}$ such that $v_{i}$ is on a cycle and has $\delta_{T\left(B_{i, 1, \ldots, k}\right)}\left(v_{i}\right)=2$. If $B_{i, 1}$ is trivial, then the result is a Christmas cactus spanning 


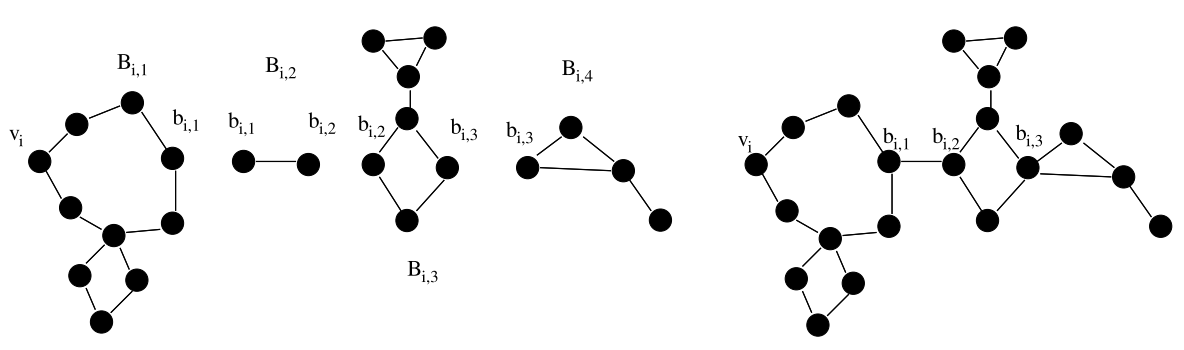

Fig. 4 Connecting spanning Christmas cactus graphs in a plane chain of blocks

subgraph for the plane chain of blocks $B_{i, 1}, b_{i, 1}, \ldots, b_{i, k-1}, B_{i, k}$ such that $v_{i}$ is not on a cycle and $\delta_{T\left(B_{i, 1, \ldots, k}\right)}\left(v_{i}\right)=1$.

Then consider the base cycle $V(C)$ and join the spanning Christmas cactus graph of each induced plane chain of blocks $V_{i} \cup\left\{v_{i}\right\}$ to the node $v_{i}$ on the cycle by an edge, and contract the edge as in Fig. 4.

The result is a spanning Christmas cactus graph of $G$ such that $x, y$ are jointly on a cycle and $\delta_{T(G)}(x), \delta_{T(G)}(y)=2$, and the theorem is true by induction. This construction also yields a polynomial-time algorithm because each decomposition is polynomial-time constructible and $x, y$ do not appear in the decomposition, and so the number of decompositions that must be computed is bounded by $\frac{n}{2}$.

\subsection{Constructing a Greedy Embedding}

In this section, we construct a greedy embedding for any Christmas cactus graph in the Euclidean plane.

Let $G$ be an arbitrary Christmas cactus graph, and let $F$ be the set of edges in $G$ that are not contained in a simple cycle. For the purposes of this construction, all edges in $F$ will be considered to be simple cycles (on two nodes). Then every edge in $G$ is contained in exactly one simple cycle.

Definition 5 A depth tree $T$ w.r.t. $G$ is a tree that contains a node for each simple cycle in $G$, where nodes in $T$ are connected iff $\left|V\left(C_{1}\right) \cap V\left(C_{2}\right)\right|=1$.

Select an arbitrary node of $T$ to serve as the "root" and define the depth of a cycle in the graph $G$ as the depth of the corresponding node in $T$. Then define the depth of any node in $G$ to be the minimum depth of any cycle containing that node. $G$ will be embedded on concentric semi-circles of radius $1=R_{0}<R_{1}<R_{2}<\cdots$ such that all nodes at depth $i$ will be embedded on the semi-circle of radius $R_{i}$. Let the center of all the semi-circles be the origin.

For any cycle $C=\left(p, x_{1}, \ldots, x_{m}\right)$ at depth $k>0$, there will be a unique node on the cycle that is at depth $k-1$, and all remaining nodes will be at depth $k$. Assume that for the cycle $C$, the unique node on the cycle that is at depth $k-1$ is $p$. Then $p$ will be embedded on the semi-circle at radius $R_{k-1}$, and node $x_{1}$ will be placed at the intersection of the semi-circle of radius $R_{k}$ and the ray that contains $p$ and the origin. The remaining nodes (if any) $x_{2}, \ldots, x_{m}$ will be embedded on the semi-circle 
a

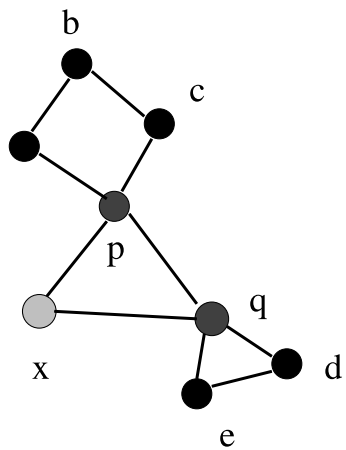

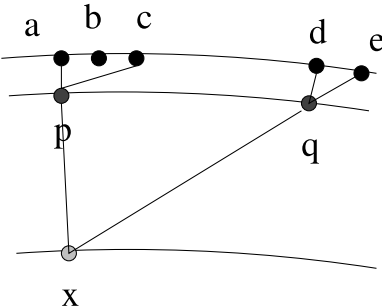

depth $\mathrm{i}+2$

depth $i+1$

depth i

Fig. 5 Embedding a Christmas cactus graph on concentric semi-circles

of radius $R_{k}$ such that the nodes $x_{1}, x_{2}, \ldots, x_{m}$ appear in clock-wise order along the semi-circle of radius $R_{k}$ at a distance to be specified shortly. See Fig. 5.

Definition 6 A node $u \in G$ is a descendant of the cycle $C$ at depth $k$ if after removing all edges in $C$ from $G$, node $u$ is not in the component that also contains node $p$, the unique node in $C$ at depth $k-1$.

A node $u$ in the cycle $C$ at depth $k$, which is not the unique node in $C$ at depth $k-1$, is also a descendant of the cycle $C$ by this definition.

The embedding will proceed in phases, and at the end of phase $i$ all nodes at depth $\leq i$ will be placed. To simplify the analysis, after each phase (and subphase), we will preserve the greedy property that the subgraph induced by all currently placed nodes along with the current embedding must exhibit the greedy routing property. Formally, if at the end of a subphase, the set of nodes in $G$ already placed is $P$ and the subgraph induced by $P$ is $G_{P}$, then for all $s, t \in P$, there exists a node $u\left(\operatorname{adjacent}\right.$ to $s$ in $G_{P}$ ) such that $d(u, t)<d(s, t)$.

We will use a geometric lemma to establish the properties needed for this embedding scheme. Consider the coordinates (assume $\epsilon>0$ and $0 \leq \alpha, \beta \leq \pi$ )

$$
\begin{aligned}
& c=(0,1+z) \\
& b=(-\sin \beta, \cos \beta) \\
& a=(-(1+\epsilon) \sin (\beta-\alpha),(1+\epsilon) \cos (\beta-\alpha))
\end{aligned}
$$

subject to the constraints

$$
\begin{aligned}
0 & <\alpha, \beta \leq \frac{\pi}{2} \\
0 & <\epsilon \leq \frac{1-\cos \beta}{6} \\
0 & \leq z \leq \epsilon \\
\sin \alpha & \leq \frac{\epsilon(1-\cos \beta)}{2(1+\epsilon)}
\end{aligned}
$$


Claim $2 \frac{d}{d z}\left(d(a, c)^{2}-d(b, c)^{2}\right)<0$.

Proof

$$
\begin{aligned}
& \frac{d}{d z}\left(d(a, c)^{2}-d(b, c)^{2}\right) \\
& \quad=2(1+z-(1+\epsilon) \cos (\beta-\alpha))-2(1+z-\cos \beta) \\
& \quad=2(\cos \beta-(1+\epsilon) \cos (\beta-\alpha)) \\
& \quad=2(\cos \beta-(1+\epsilon)(\cos \beta \cos \alpha+\sin \beta \sin \alpha)) \\
& \quad \leq 2 \cos \beta(1-(1+\epsilon) \cos \alpha) \\
& \quad<0
\end{aligned}
$$

where the last inequality follows because $\sin \alpha \leq \frac{\epsilon}{1+\epsilon}$ and $\cos \alpha \geq 1-\frac{\epsilon^{2}}{(1+\epsilon)^{2}}=$ $\frac{1+2 \epsilon}{(1+\epsilon)^{2}}>\frac{1}{1+\epsilon}$.

Hence $d(a, c)^{2}-d(b, c)^{2}$ is minimized for $z=\epsilon$.

Claim $3 d(a, c)-d(b, c) \geq \epsilon^{2}$.

Proof By Claim 1,

$$
\begin{aligned}
& d(a, c)^{2}-d(b, c)^{2} \\
& \geq(1+\epsilon)^{2} \sin ^{2}(\beta-\alpha)-\sin ^{2} \beta \\
&+(1+\epsilon)^{2}(1-\cos (\beta-\alpha))^{2}-(1+\epsilon-\cos \beta)^{2} \\
& \geq(1+\epsilon)^{2} \sin ^{2}(\beta-\alpha)-2(1+\epsilon)(1-\cos \beta) \\
&+(1+\epsilon)^{2}(1-\cos (\beta-\alpha))^{2}-\epsilon^{2} \\
&= 2(1+\epsilon)^{2}(1-\cos (\beta-\alpha))-2(1+\epsilon)(1-\cos \beta)-\epsilon^{2} \\
&=-\epsilon^{2}+2(1+\epsilon)(\epsilon+\cos \beta-(1+\epsilon) \cos (\beta-\alpha)) \\
& \geq-\epsilon^{2}+2(1+\epsilon)(\epsilon+\cos \beta-(1+\epsilon)(\cos \beta+\sin \alpha)) \\
&=-\epsilon^{2}+2(1+\epsilon)(\epsilon(1-\cos \beta)-(1+\epsilon) \sin \alpha) \\
& \geq-\epsilon^{2}+(1+\epsilon) \epsilon(1-\cos \beta) \\
& \geq 5 \epsilon^{2}
\end{aligned}
$$

Then

$$
\begin{aligned}
d(a, c)-d(b, c) & =\frac{d(a, c)^{2}-d(b, c)^{2}}{d(a, c)+d(b, c)} \\
& \geq \frac{1}{5}\left(d(a, c)^{2}-d(b, c)^{2}\right) \\
& \geq \epsilon^{2}
\end{aligned}
$$

since $d(a, c)+d(b, c) \leq 5$. 
For notational convenience, given an embedding $f: V \rightarrow R^{2}$, define the angle $\angle a, b, c$ on nodes $a, b, c, \in V(G)$ as the angle formed by the rays $(f(b), f(a))$ and $(f(b), f(c))$.

Theorem 3 For any Christmas cactus graph $G$, there exists a greedy embedding of $G$ into the Euclidean plane.

Proof Assume that every edge is in a simple cycle by considering any edge not in a simple cycle as a 2 -cycle. Construct the depth tree $T$ w.r.t. $G$, and root $T$ at an arbitrary node. Trace out a semi-circle of radius 1 centered at the origin. Suppose that the cycle $C$ in $G$ at depth 0 contains $m$ nodes, $C=(1,2, \ldots, m)$. Then divide the semicircle of radius $R_{0}$ into $m$ equal angle sectors and place node $i$ at the beginning of the $i$ th sector. The arc subtended by $1,2, \ldots, m$ is strictly smaller than the perimeter of the semi-circle of radius $R_{0}=1$ because no node is placed at the end of the $m$ th sector.

For any triple $(a, a+1, c)$ such that $c>a$, the angle $\angle a, a+1, c$ is strictly larger than $\frac{\pi}{2}$ and $d(a+1, c)<d(a, c)$. Similarly, for any triple $(a, c-1, c)$ such that $c>a$, the angle $\angle a, c-1, c$ is strictly larger than $\frac{\pi}{2}$ and $d(a, c-1)<d(a, c)$. Hence this embedding is greedy.

This establishes the base case for the inductive construction. Now assume that all nodes at depth $\leq i$ have been placed and that the induced subgraph on these nodes, $G_{i}$, along with the embedding on concentric semi-circles (as described earlier) is greedy.

Definition 7 If all nodes in $G_{i}$ have been embedded so that this embedding exhibits the greedy property, then for all $s, t \in G_{i}$, there exists $u \in G_{i}$ such that $(u, s) \in E\left(G_{i}\right)$ and $d(u, t)<d(s, t)$. Fix $n_{s, t}=u$ and define $\delta\left(G_{i}\right)=\min _{s, t} d(s, t)-d\left(t, n_{s, t}\right)$.

Then draw a ball $B_{u}$ of radius $\delta\left(G_{i}\right) / 3$ around each node $u \in G_{i}$. Clearly if a node $t$ at depth $i+1$ such that $(u, t) \in E(G)$ is placed in $B_{u}$, then for any node $s \in G_{i}, \neq t$, the neighbor $n_{s, t}$ that is strictly closer to $t$ will also be strictly closer to $u$. And if $s=t \in G_{i}$, then the neighbor $u$ will be strictly closer to $u$.

Definition 8 Let $\beta\left(G_{i}\right)$ be defined as the minimum (nonzero) angle over all $s, t$ at depth $\leq i$ from $s$ to the origin, to $t$ in the current embedding.

Assume that all nodes at depth $\leq i$ have been placed and that the subgraph induced by these nodes along with the embedding exhibits the greedy property. We must embed all cycles at depth $i+1$ and preserve the greedy property.

Subphase: For each cycle $C$ at depth $i+1, C=\left(p, x_{1}, \ldots, x_{m}\right)$, let $p$ be the unique node in the cycle at depth $i$. Call $x_{1}$ the representative node for the cycle $C$ (choose an orientation of $C$ at random and choose the next node after $p$ ). Let the radius of the outermost semi-circle in the current embedding be $R_{i}$ and define $\delta\left(G_{i}\right)$ and $\beta\left(G_{i}\right)$ as before w.r.t. the current embedding. Also, define $\epsilon=\min \left(\frac{\delta\left(G_{i}\right)}{3}, R_{i} \frac{1-\cos \frac{2}{3} \beta\left(G_{i}\right)}{6}\right)$.

Place each representative node $x_{1}$ at the intersection of the semi-circle of radius $R_{i+1}=R_{i}+\epsilon$ and the ray containing both the origin and $p$. Let $P$ be the set 
currently placed nodes (all nodes at depth $\leq i$ and one representative node for each cycle at depth $i+1)$. We will show that the subgraph induced by these nodes, along with the current embedding, exhibits the greedy property:

For any nodes $s, t \in G_{i}$, there is trivially a node $u$ (adjacent to $s$ in $G_{i}$ ) in the current embedding such that $d(u, t)<d(s, t)$ because the nodes in $G_{i}$ along with the embedding of these nodes exhibited the greedy property (and the embedding of nodes in $G_{i}$ has not been changed) by induction. For any nodes $s, t$ such that $s \in G_{i}$ and $t$ is a representative node for a cycle $C$ at depth $i+1$, we have that $t$ is in the ball $B_{p}$ of radius $\frac{\delta\left(G_{i}\right)}{3}$ centered around the node $p$ in $C$ that is the unique node in $C$ at depth $i$. Then $(t, p)$ is an edge in $G$, and all $s \in G_{i}$ have a (already placed) neighbor that is strictly closer to $t$.

Lastly, consider routing from a node $s$ that is a representative node on a cycle $C$ at depth $i+1$ to any node that has already been placed. Again, let $p$ be the unique node in $C$ that is at depth $i$. By construction, the perpendicular bisector to the segment $s p$ contains all nodes currently placed, except $s$, on the same side as $p$ :

Trivially, any node $t$ at depth $\leq i$ will be on the same side of the perpendicular bisector to $s p$ as the node $p$ because the perpendicular bisector is a parallel shift of the tangent to the semi-circle of radius $R_{i}$ at the point $p$. And for any node $t$ that is placed on the semi-circle at radius $R_{i+1}$, the angle from $t$ to the origin to $s$ will be at least $\beta\left(G_{i}\right)$, and by the geometric lemma (choosing $\alpha=0$ ), this node will also be on the $p$ side of the perpendicular bisector to $s p$. Thus $p$ is strictly closer to $t$ than $s$ is to $t$ for any node $t$ at depth $i+1$ that has been placed in this subphase.

All cases are covered: the subgraph on the currently embedded nodes, along with the current embedding, is greedy because for all $s, t$, there is an already placed neighbor of $s$ that is strictly closer to $t$.

Subphase: For a cycle $C=\left(p, x_{1}, \ldots, x_{m}\right)$ at depth $i+1$, only the unique node $p$ at depth $i$ and the representative node $x_{1}$ have been embedded so far. The embedding must now be extended to include all nodes at depth $i+1$, and this is done by placing nodes $x_{2}, \ldots, x_{m}$ in clockwise order around the semi-circle of radius $R_{i+1}$ starting from the location of node $x_{1}$.

Let $G_{i}^{1}$ be the subgraph induced by all nodes already placed. Define $\delta\left(G_{i}^{1}\right)$ as before. Note that this difference is now defined over all $s, t$ that have already been placed, which includes all nodes that are at depth $\leq i$ and all nodes that are depth $i+1$ that are representative nodes for a cycle at depth $i+1$. Also define $\epsilon=R_{i+1}-R_{i}$ and note that $\beta\left(G_{i}^{1}\right)=\beta\left(G_{i}\right)$.

Then place nodes $x_{2}, \ldots, x_{m}$ on the semi-circle of radius $R_{i+1}$ at even intervals starting from the (already fixed) location of $x_{1}$ such that the angle (in radians) from $x_{1}$ to the origin to $x_{m}$ is $\alpha \leq \min \left(\frac{\delta\left(G_{i}^{1}\right)}{3}, \frac{\beta\left(G_{i}\right)}{3}\right)$ and such that $\sin \alpha \leq \frac{\epsilon\left(1-\cos \frac{2}{3} \beta\left(G_{i}\right)\right)}{2(1+\epsilon)}$. Place all nodes on a cycle at depth $i+1$ that have not already been placed, according to this rule. The induced subgraph on all placed nodes after this subphase, along with the embedding, will exhibit the greedy property:

Now all nodes at depth $\leq i+1$ have been placed. Consider all pairs $s, t \in G_{i+1}$. Again, if $s, t \in G_{i}^{1}$, then there will still be a neighbor of $s$ that is strictly closer.

If $s \in G_{i}^{1}$, and $t$ is placed in this subphase, then $t$ is at most distance $\frac{\delta\left(G_{i}^{1}\right)}{3}$ from the representative node $u$ on the same cycle. $s \neq u$ will have a neighbor that is strictly closer to this representative node $u$, and this same neighbor will also be strictly closer 
to $t$. If $s=u$, then $s$ and $t$ will be connected by a path on the semi-circle of radius $R_{i+1}$, and each successive node on this path will be strictly closer to $t$.

The only remaining case is where $s$ is a node that is placed in this subphase:

Any node that is not in the set $x_{2}, x_{3}, \ldots, x_{m}$ cannot be strictly contained in the sector from $x_{1}$ to the origin to $x_{m}$ because we chose $\alpha \leq \frac{\beta\left(G_{i}\right)}{3}$. Then if $s$ is not $x_{1}$ or $x_{m}, s$ will have a neighbor that is strictly closer to $t$, choosing the next node on the path $x_{1}, x_{2}, \ldots, x_{m}$ radially in the direction of $t$.

The case in which $s=x_{1}$ has already been covered because the node $x_{1} \in G_{i}^{1}$. Then suppose $s=x_{m}$. If $t$ is in the set $x_{1}, \ldots, x_{m}$, then choosing the next node on the path $x_{1}, x_{2}, \ldots, x_{m}$ radially in the direction of $t$ and $s$ will have a neighbor strictly closer to $t$. And if $t$ is not in the set $x_{1}, \ldots, x_{m}$, then $\alpha \leq \frac{1}{3} \beta\left(G_{i}\right)$, and this implies that the angle from $p$ to the origin to the node $t$ is at least $\frac{2}{3} \beta\left(G_{i}\right)$. By the geometric lemma, $d\left(x_{m}, t\right)-d(p, t)>0$, and $p$ is strictly closer to $t$.

Then all cases are covered, and the subgraph on the currently embedded nodes, along with the current embedding, is greedy because for all $s, t$, there is an already placed node that is strictly closer to $t$.

This completes the inductive construction, because all nodes at depth $\leq i+1$ have been placed. This also completes the proof that all Christmas cactus graphs can be greedily embedded in the Euclidean plane.

Corollary 1 Any 3-connected graph $G$ that excludes $K_{3,3}$ as a minor admits a greedy embedding into the Euclidean plane.

We can apply a theorem in [9] which states that if a 3-connected graph $G$ does not contain $K_{3,3}$ as a minor, then $G$ contains a spanning 3-connected planar subgraph. Using the results of this section, this immediately implies the corollary.

\section{Greedy Embeddings for Trees}

In this section, we give the first examples of graphs of degree at most 3 that admit no greedy embedding into the Euclidean plane. We also prove a combinatorial condition that guarantees nonembeddability, and this condition provides a certificate for nonembeddability that can be verified in linear time. We use this result to construct graphs that can be greedily embedded into the Euclidean plane, but for which no spanning tree admits such an embedding.

Barnette [1] proved that all 3-connected planar graphs contain a spanning tree of maximum degree at most 3 . Given any spanning closed 2 -walk, we can construct a spanning tree of maximum degree at most 3 using only edges traversed by such a walk. So Barnette's Theorem is implied by the result of Gao and Richter [5]. We showed that a particular type of spanning closed 2-walk-a spanning Christmas cactus graph-is enough to construct a greedy embedding into the Euclidean plane. And as a corollary to the results we present in this section, a sufficiently large complete binary tree admits no greedy embedding into the Euclidean plane. So, the results due to Gao and Richter are almost enough to guarantee a greedy embedding into the Euclidean plane, but the results of Barnette [1] are not sufficient. 


\subsection{Irreducible Triples}

Definition 9 An irreducible triple is a triple $\{b, c, d\}$ of nodes in a graph $G$ such that $\operatorname{deg}(b)=3$ and $(b, c),(b, d) \in E(G)$ and removing either $(b, c)$ or $(b, d)$ disconnects the graph. The parent of an irreducible triple $\{b, c, d\}$ is the unique node $a \notin\{b, c, d\}$ such that $(a, b) \in E(G)$.

Definition 10 Two irreducible triples $\{b, c, d\}$ and $\{x, y, z\}$ are said to be independent if $\{b, c, d\} \cap\{x, y, z\}=\emptyset$ and if deleting edges $(b, c),(b, d),(x, y)$, and $(x, z)$ leaves $b$ and $x$ connected. A set of irreducible triples is mutually independent if the irreducible triples are pairwise independent.

Lemma 2 Consider any set of three or more mutually independent irreducible triples $\Lambda$. Let $a$ be the parent of any irreducible triple in the set. Then for all irreducible triples $\{x, y, z\}$ in the set $\Lambda,\{a\} \cap\{x, y, z\}=\emptyset$ (including the triple for which $a$ is the parent).

Proof Clearly any parent $a$ of an irreducible triple $\{b, c, d\}$ cannot intersect $\{b, c, d\}$ directly from the definition of parent. Suppose that there is a set of three or more mutually independent triples in a graph $G$ and that the parent $a$ of an irreducible triple $\{b, c, d\}$ is contained in another irreducible triple $\{x, y, z\}$. Suppose that $a=y$. Then deleting the edge $(x, y)$ disconnects $G$, but $b$ is still connected to $y$. This implies that $b$ is not still connected to $x$. This contradicts the definition of independence.

Suppose that $a=x$. Then $\Gamma(b)=\{x, c, d\}$ and $\Gamma(x)=\{b, y, z\}$ because $x$ is the parent of the triple $\{b, c, d\}$ and $b$ is the parent of the triple $\{x, y, z\}$. Then consider a third irreducible triple in the set of three or more mutually independent irreducible triples, $\{l, m, n\}$.

By the definition of an irreducible triple, deleting the edge $(b, c)$ must partition $G$ into components $C_{1}, C_{2}$. Deleting the edge $(b, d)$ also partitions $G$ into two components. Note that $(b, d)$ cannot connect $C_{1}$ and $C_{2}$ that resulted from deleting $(b, c)$. Then deleting $(b, d)$ after deleting $(b, c)$ must partition $G$ into three components, one of which contains $b$, one of which contains $c$, and one of which contains $d$. Continuing the argument, deleting edges $(b, c),(b, d),(x, y)$, and $(x, z)$ partitions $G$ into five components, one of which contains $b$ and $x$, one of which contains $c$, one of which contains $d$, one of which contains $y$, and one of which contains $z$. The component that contains $b$ and $x$ contains only the nodes $b$ and $x$, because $\operatorname{deg}(b)=\operatorname{deg}(x)=3$ before deleting two of the edges incident to $b$ and two of the edges incident to $x$.

Node $l$ must be contained in a different component than $b$ and $x$. Let this component be the component that contains $y$. Then $\{l, m, n\}$ would not be independent from $\{x, y, z\}$ because deleting the edge $(x, y)$ from $G$ would leave $l$ and $x$ in different components, and this contradicts the definition of independence.

Thus if there is a set of three or more mutually independent irreducible triples, then the parent $a$ of any irreducible triple $\{b, c, d\}$ in the set cannot be contained in any other triple in the set.

Lemma 3 For any set of three or more mutually independent triples, let $\{b, c, d\}$ and $\{x, y, z\}$ be two irreducible triples in the set, and let $a, w$ be the respective 
parents of these irreducible triples (note that a and $w$ are not guaranteed to be distinct). Then any simple path from $y$ to $c$ in the graph $G$ must be of the form $(y, x),(x, w), \ldots,(a, b),(b, c)$. Any simple path from $x$ to $c$ must be of the form $(x, w), \ldots,(a, b),(b, c)$. And any simple path from $x$ to $b$ must be of the form $(x, w), \ldots,(a, b)$.

Proof The proof immediately follows from the previous lemma.

We will implicitly use the path lemma throughout the proof that any graph $G$ containing a set of six or more mutually independent irreducible triples cannot be greedily embedded. Let $S=\cup\{b, c, d\}$ be the set of all nodes in any irreducible triple in the set. Let $\{b, c, d\}$ be a particular irreducible triple in the set, and let $a$ be the parent of this triple. Suppose that $G$ admits a greedy embedding $f: V \rightarrow R^{2}$. Consider the halfspace $H_{b}$ that is bounded by the perpendicular bisector to $f(b), f(c)$ that contains $f(b)$. This halfspace must contain $S /\{c\}$ from the path lemma, because the only simple paths from $c$ to a node $t \in S /\{c\}$ must begin by traversing the edge $(c, b)$. Similarly, the halfspace $H_{a}$ that is bounded by the perpendicular bisector to $f(a), f(b)$ and contains $f(a)$ must contain $S /\{b, c, d\}$ again from the path lemma.

Lemma 4 Let $G$ be a graph that admits a greedy embedding $f: V \rightarrow R^{2}$ into the Euclidean plane, and let $(p, x) \in E(G)$ be an edge such that deleting $(p, x)$ disconnects $G$. Let $C_{x}$ be the component containing $x$ that results from deleting the edge $(p, x)$, and let $z$ be an arbitrary node $\notin C_{x}$. Then $\{x\}=W_{z}=\arg \min _{w \in C_{x}} \| f(w)-$ $f(z) \|_{2}$.

Proof Suppose that $W_{z} \neq\{x\}$ and there is a node $w \in \arg \min _{w \in C_{x}}\|f(w)-f(z)\|_{2}$ such that $w \neq x$. All the neighbors of $w$ are in $C_{x}$, and no node in $C_{x}$ is strictly closer to $z$. Then there is no neighbor of $w$ that is strictly closer to $z$, and $f$ is not a greedy embedding.

Lemma 5 Let $G$ be a graph that admits a greedy embedding $f: V \rightarrow R^{2}$ into the Euclidean plane and that contains an irreducible triple $\{b, c, d\}$. Then any greedy embedding into the Euclidean plane must map the nodes $b, c, d$ to points in $R^{2}$ such that the angle $\angle f(c) f(b) f(d)>\frac{\pi}{3}$.

Proof Suppose that the angle $\angle f(c) f(b) f(d)$ is $\leq \frac{\pi}{3}$. Then by the law of sines, the side $(c, d)$ cannot be the strictly largest side in the triangle $(c, b, d)$. Let $(b, d)$ be the largest side in the triangle. Node $d \notin C_{c}$, and $c$ must be the closest node in $C_{c}$ (the component that results from deleting the edge $(b, c))$ to $d$. When routing from node $c$ to node $d$, node $b$ must be selected for the next hop. However $d(b, d) \geq d(c, d)$, and this embedding cannot be greedy.

Claim 4 Any graph that contains two independent irreducible triples $\{b, c, d\}$ and $\{x, y, z\}$-where $\Gamma(b)=\{a, c, d\}, \Gamma(x)=\{w, y, z\}$-cannot be greedily embedded so that $f(a)$ is contained in a side of the angle $\angle f(c) f(b) f(d)$ that is $\leq \pi$ and $f(w)$ is contained in a side of the angle $\angle f(y) f(x) f(z)$ that is $\leq \pi$. 
Fig. 6 Embedding a quadruple $\{a, b, c, d\}$ such that $f(a)$ is contained in a side of the angle $\angle f(c) f(b) f(d)$ that is $<\pi$

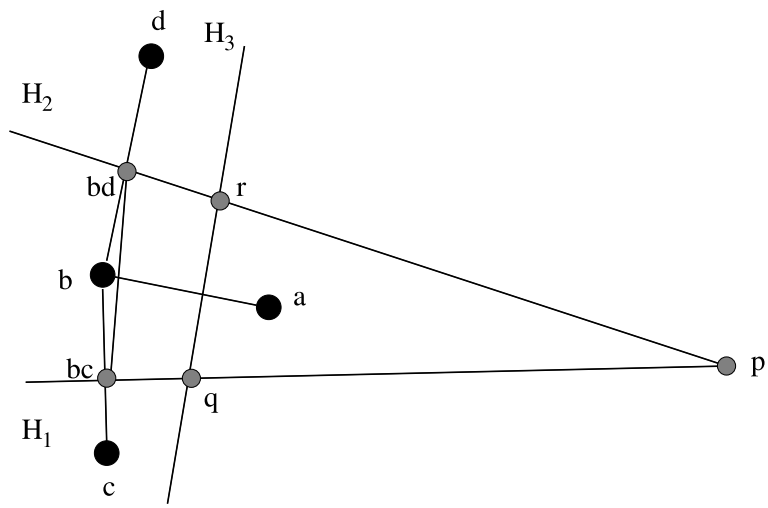

Proof Assume that both $\angle f(c) f(b) f(d)$ and $\angle f(y) f(x) f(z) \neq \pi$. Consider a greedy embedding of the quadruple $\{a, b, c, d\}$ depicted in Fig. 6.

If the embedding is greedy, then there must be a path from $a$ to $c$ such that the distances to the destination node, $c$, are strictly decreasing along this path. There must also be such a path from $a$ to $d$. Any such path contains $b$ as an intermediary node, and this implies that $d(f(b), f(c))<d(f(a), f(c))$ and $d(f(b), f(d))<$ $d(f(a), f(d))$. This implies that $f(c)$ and $f(d)$ must be contained on the $b$ side of the line $\mathrm{H}_{3}$. As a result, the segment $(b c, b d)$ must be contained on the $b$ side of $\mathrm{H}_{3}$ because the line segment is contained in the convex hull of the points $f(c), f(d), f(b)$.

Using a similar argument, all nodes in $G$ not in $\{b\} \cup C_{c} \cup C_{d}$ must be strictly contained in the triangle $(p, q, r)$, because all nodes in $G$ not in $\{b\} \cup C_{c} \cup C_{d}$ must be strictly on the $b$ side of $H_{1}$, strictly on the $b$ side of $H_{2}$ and strictly on the $a$ side of $H_{3}$, respectively. Because the segment $(b c, b d)$ is contained on the $b$ side of $H_{3}$, we can relax this constraint to the requirement that all nodes in $G$ not in $\{b\} \cup C_{c} \cup C_{d}$ must be strictly contained in the triangle $(b c, b d, p)$.

An identical argument holds for the quadruple $\{w, x, y, z\}$, and all nodes in $G$ not in $\{x\} \cup C_{y} \cup C_{z}$ must be strictly contained in the triangle $(x y, x z, o)$.

Consider the point $b c$. This lies in the convex hull of $f(b), f(c)$, and any convex body (specifically the triangle $(x y, x z, o)$ ) that strictly contains $f(b)$ and $f(c)$ must strictly contain $b c$. Similarly the point $b d$ must be strictly contained in the triangle $(x y, x z, o)$. This implies that the segment $(b c, b d)$ must be strictly contained in the triangle $(x y, x z, o)$. An identical argument holds for the triangle $(b c, b d, p)$, and this triangle must strictly contain the segment $(x y, x z)$.

However, this yields a contradiction because there are two triangles $T_{1}$ and $T_{2}$ such that $T_{1}$ must strictly contain the base of $T_{2}$ and $T_{2}$ must strictly contain the base of $T_{1}$. An almost identical argument holds when $\angle f(c) f(b) f(d)=\pi$ or $\angle f(y) f(x) f(z)=\pi$.

Claim 5 If a graph $G$ is greedily embedded and contains an irreducible triple $\{b, c, d\}$-where $\Gamma(b)=\{a, c, d\}$-that is embedded so that $f(a)$ is contained in a side of the angle $\angle f(c) f(b) f(d)$ that is $>\pi$, then let $i$ be the point of intersection of the perpendicular bisector to the segment $(f(b), f(c))$ and the perpendicular bi- 
Fig. 7 Embedding a quadruple $\{a, b, c, d\}$ such that $f(a)$ is contained in a side of the angle $\angle f(c) f(b) f(d)$ that is $>\pi$

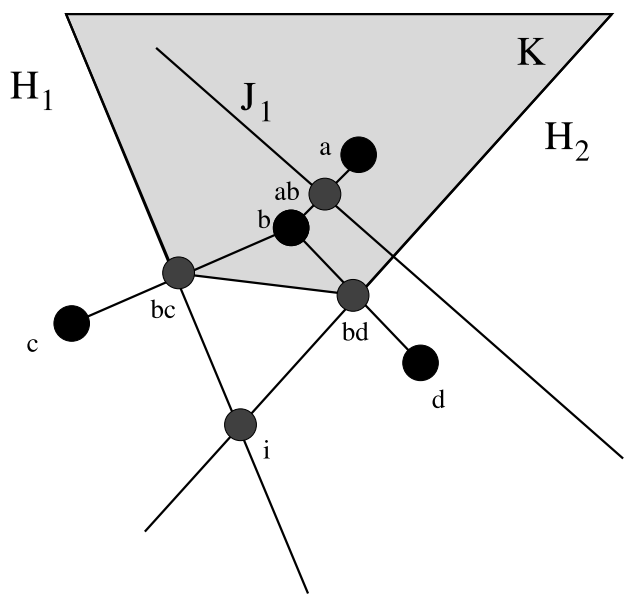

sector to the segment $(f(b), f(d))$. All nodes not in $C_{c} \cup C_{d}$ are mapped outside the interior of the quadrilateral $(f(c), f(b), f(d), i)$.

Proof Consider Fig. 7. Clearly, we must only prove that all nodes not in $C_{c} \cup C_{d}$ are mapped outside the quadrilateral $(b c, f(b), b d, i)$ because the line $H_{1}$ must contain all nodes not in $C_{c}$ on the $b$ side, and the line $H_{2}$ must contain all nodes not in $C_{d}$ on the $b$ side.

Consider the halfspace $J_{1}$ defined as the $a$ side of the perpendicular bisector to $f(a), f(b)$. Node $a$ is not mapped into the quadrilateral $(b c, f(b), b d, i)$ by assumption, and any node in $G$ not in $\{b\} \cup C_{c} \cup C_{d}$ must be contained in the halfspace $J_{1}$. If the intersection of $J_{1}$ with the quadrilateral $(b c, f(b), b d, i)$ is empty, then clearly the claim is proven. The halfspace $J_{1}$ has an empty intersection with the triangle $(f(b), b c, b d)$ because the points $f(b), f(c), f(d)$ must be on the $b$ side of the perpendicular bisector to $(f(a), f(b))$.

Consider the triangle $(b c, b d, i)$. Assume that $J_{1}$ intersects the triangle $(b c, b d, i)$. Then $J_{1}$ must contain at least one of the points $b c, b d, i . J_{1}$ cannot contain $b c$ or $b d$ because $f(b), f(c)$, and $f(d)$ must all be closer to $f(b)$ than to $f(a)$. As a result, if $J_{1}$ intersects the triangle $(b c, b d, i)$, then $J_{1}$ must contain the point $i$.

A line can intersect another line more than once only if the two lines are identical. Consider the line $L$ bounding the halfspace $J_{1}$. Suppose that this line is identical to $H_{1}$. This can only happen if $f(a)=f(c)$, and this would imply that the embedding is not greedy because node $a$ will not have a neighbor that is strictly closer to $f(c)$. This implies that the line $L$ can intersect $H_{1}$ and $H_{2}$ at most once each.

$J_{1}$ contains the point $i$ but either of the points $b c$ or $b d$. This implies that the line $L$ intersects both segments $(b c, i)$ and $(b d, i)$. This line can be cut into two rays, leaving from the point $a b$ in opposite directions. Both rays begin at the point $a b$ inside the shaded region $K$ and cannot leave this region through the segment $(b c, b d)$ because both end points of this segment are not contained in $J_{1}$.

Then one of the rays must exit the region $K$ through a side bounded by either the line $H_{1}$ or the line $H_{2}$. This yields a contradiction because the line $L$ will intersect 

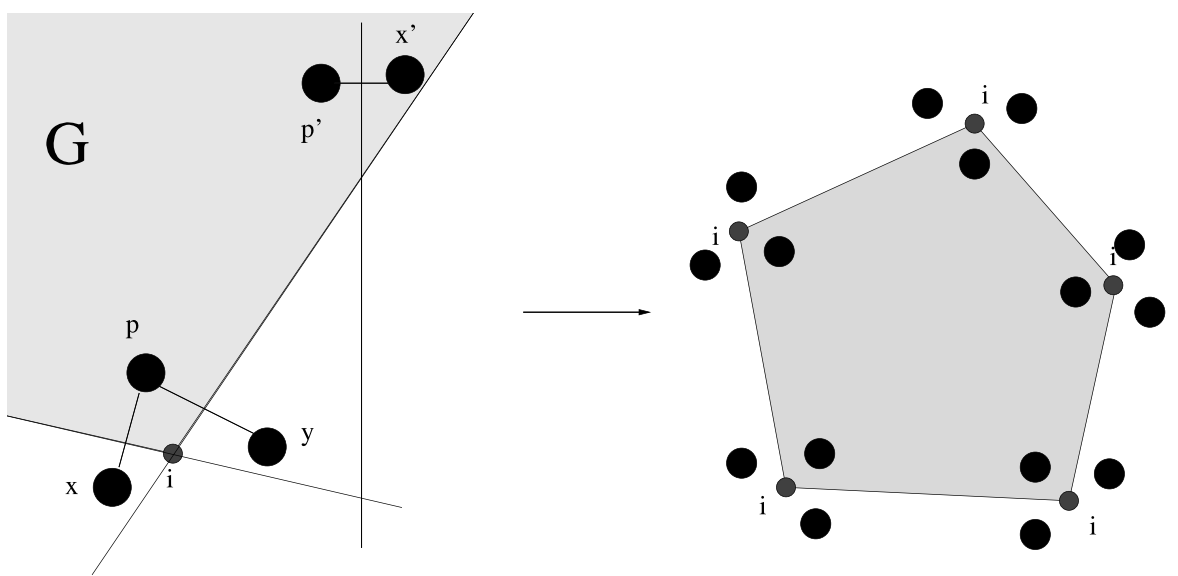

Fig. 8 Embedding quadruples

either the line $H_{1}$ or the line $H_{2}$ twice. Thus $J_{1}$ cannot contain $i$, and the claim is proven.

Note that $\angle b c i b d<\frac{2 \pi}{3}$ because $\angle b c f(b) b d>\frac{\pi}{3}$ and $\angle f(b) b c i=\angle f(b) b d i=$ $\frac{\pi}{2}$. When a point $x$ is contained in the sector $\angle b c i b d$, we will say the intersection point $i$ contains $x$.

Lemma 6 Any graph $G$ containing six or more mutually independent irreducible triples cannot be greedily embedded in the Euclidean plane.

Proof Suppose that a graph $G$ contains six mutually independent irreducible triples. Then there are two cases to consider:

Suppose that the irreducible triples are embedded so that for each quadruple $\{a, b, c, d\}$-where $\{b, c, d\}$ is an irreducible triple and $\Gamma(b)=\{a, c, d\}-f(a)$ is contained in a side of the angle $\angle f(c) f(b) f(d)$ that is $>\pi$. Then for each quadruple $\{a, b, c, d\}$, define the points $b c, b d, i$ as in Fig. 7 .

No node in $G$ is mapped to a point in the triangle $(b c, b d, i)$ from the previous claim. Then consider another quadruple $\{w, x, y, z\}$, where $\{x, y, z\}$ is an irreducible triple and $\Gamma(x)=\{w, y, z\}$. Reusing the argument used in the previous claim, if the perpendicular bisector $L$ to the segment $(f(x), f(y))$ does not contain $i$ on the $x$ side, then $L$ must intersect either $H_{1}$ or $H_{2}$ twice. But $L$ is a perpendicular bisector to two points that are contained on the same side of $H_{1}$ and on the same side of $H_{2}$, and $L$ cannot be identical to $H_{1}$ or $H_{2}$.

Then defining the intersection point $i$ for each quadruple $\{a, b, c, d\}$ as in Fig. 7, each intersection point must contain all other intersection points in an angle that is $<\frac{2 \pi}{3}$. Define the polygon on these intersection points as in Fig. 8-all angles in the polygon are $<\frac{2 \pi}{3}$. By elementary geometry, any polygon on $n \geq 6$ nodes must contain an angle that is at least $\frac{2 \pi}{3}$. Then there can be at most five intersection points. This yields a contradiction, because there are six intersection points. 
Fig. 9 A graph that admits a greedy embedding into the Euclidean plane, and yet no spanning tree of this graph admits such an embedding

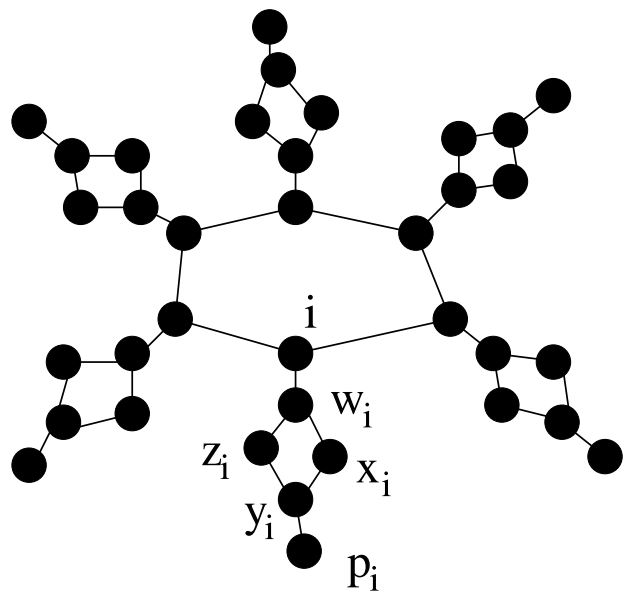

Suppose that one quadruple $\{a, b, c, d\}$ is embedded so that $f(a)$ is contained on the side of the angle $\angle f(c) f(b) f(d)$ that is $\leq \pi$. Consider the points $b c, b d$, and define intersection points for all remaining irreducible triples. Then each intersection point for the remaining five irreducible triples must contain $b c$ and $b d$ because these intersection points must contain $f(b), f(c)$, and $f(d)$. We can apply the argument used above to the perpendicular bisectors $H_{1}, H_{2}$, and $H_{3}$, and this implies that the angles bounded by $H_{1}, H_{3}$ and $H_{2}, H_{3}$ must contain each intersection point defined for the remaining five irreducible triples.

The angles bounded by $H_{1}, H_{3}$ and $H_{2}, H_{3}$ sum to at most $\pi$, and using the five intersection points and the points $b c, b d$, we have a polygon on seven nodes such that the angles sum to at most $\frac{13}{3} \pi$, which yields a contradiction because the sum of the angles in a seven-gon is $5 \pi$ by elementary geometry.

Corollary 2 The complete binary tree $B_{31}$ with 31 nodes cannot be greedily embedded into the Euclidean plane.

Proof The complete binary tree $B_{31}$ contains six mutually independent irreducible triples.

Theorem 4 There exist graphs that can be greedily embedded into the Euclidean plane, but for which no spanning tree can be greedily embedded into the Euclidean plane.

Proof Let $G$ be the cycle graph on $n$ nodes, and for each node $i$, add a 4-cycle $\left(w_{i}, x_{i}\right),\left(x_{i}, y_{i}\right),\left(y_{i}, z_{i}\right),\left(z_{i}, w_{i}\right)$ and an extra node $p_{i}$ such that $w_{i}$ is joined by an edge to $i$, and $p_{i}$ is joined to the node $y_{i}$. See Fig. 9. Any spanning tree of this graph contains $n$ mutually independent irreducible triples; however, this graph is a Christmas cactus graph and can be greedily embedded into the Euclidean plane.

Acknowledgements We would like to thank Robert Kleinberg for introducing us to this problem and for many helpful discussions. We would also like to thank an anonymous reviewer for many helpful comments. We would also like to thank anonymous referees for their help in improving this paper. 


\section{References}

1. Barnette, D.: Trees in polyhedral graphs. Can. J. Math. 18, 731-736 (1966)

2. Bose, P., Morin, P., Stojmenovic, I., Urrutia, J.: Routing with guaranteed delivery in ad hoc wireless networks. Wirel. Netw. 7, 609-616 (2001)

3. Dhandapani, R.: Greedy drawings of triangulations. In: Symposium on Discrete Algorithms, pp. 102111 (2008)

4. Eppstein, D., Goodrich, M.T.: Succinct greedy graph drawing in the hyperbolic plane. In: Graph Drawing, pp. 14-25 (2008)

5. Gao, Z., Richter, R.: 2-walks in circuit graphs. J. Comb. Theory Ser. B 62, 259-267 (1994)

6. Goodrich, M.T., Strash, D.: Succinct greedy geometric routings in $\mathfrak{R}^{2}$. In: CoRR (2008)

7. Karp, B., Kung, H.T.: GPSR: greedy perimeter stateless routing for wireless networks. In: Proceedings of the 6th Annual International Conference on Mobile Computing and Networking, pp. 243-254 (2000)

8. Kleinberg, R.: Geographic routing in hyperbolic space. In: INFOCOM 2007, 26th IEEE Conference on Computer Communications, pp. 1902-1909 (2007)

9. Papadimitriou, C., Ratajczak, D.: On a conjecture related to geometric routing. Theor. Comput. Sci. 244, 3-14 (2005)

10. Rao, A., Papadimitriou, C., Shenker, S., Stoica, I.: Geographic routing without location information. In: Proceedings of the 9th Annual International Conference on Mobile Computing and Networking, pp. 96-108 (2003) 